\title{
Contribuições de um Objeto de Aprendizagem Colaborativa no Contexto da CSCL
}

\author{
Eliana Cláudia Mayumi Ishikawa - UTFPR - eishikawa@utfpr.edu.br \\ Simone Nasser Matos - UTFPR - snasser@utfpr.edu.br \\ Ezequiel Gueiber - UEPG - egueiber@gmail.com \\ Guataçara dos Santos Junior - UTFPR - guata@utfpr.edu.br
}

RESUMO: Este artigo apresenta as contribuições que o objeto virtual de aprendizagem colaborativa, denominado Collabora, pode trazer no processo de ensino e aprendizagem aplicado na disciplina de Probabilidade e Estatística no Ensino Superior. A base para a análise foi obtida por meio dos registros dos alunos no chat do Collabora, das observações e anotações realizadas durante as aulas e nas atividades presenciais (diário de bordo e atividades presenciais em grupo). Para análise dos dados coletados utilizouse a Análise Textual Discursiva (ATD) que apoia o processo de categorização e unitarização. Foi criada a categoria Aprendizagem Colaborativa que contempla as áreas-chaves da CSCL e concluiu-se que o objeto educacional as atende de forma satisfatória.

Palavras-chave: Objeto de Aprendizagem. CSCL. Análise Textual Discursiva.

ABSTRACT: This article shows the contributions that the virtual object of collaborative learning, called Collabora, can bring for the teaching and learning process in the subject of Probability and Statistics in Higher Education. The basis for the analysis was obtained through the students' records in the Collabora chat room, the observations and notes made during the classes and in the classroom activities (logbook and face-to-face group activities). For the analysis of the collected data, it was used the Discursive Textual Analysis to support the categorization and unitarization process. The category Collaborative Learning were created and was concluded that the proposed educational object served CSCL's main key áreas satisfactorily. Keywords: Learning Object. CSCL. Discursive Textual Analysis.

\section{Introdução}

A Aprendizagem Colaborativa Apoiada por Computador (CSCL) tem como um dos objetivos estudar como as pessoas podem aprender em grupo com o auxílio do computador, aliada a um artefato que permite a interação entre entidades digitais tal como os Objetos de Aprendizagem (Gomes et al., 2009).

Ferramentas fundamentadas na CSCL podem ser usadas como instrumentos de trabalho que favorecem debates, confronto de ideias, troca de experiências, informações e conhecimentos, tendo em vista a construção e a reconstrução de novos conhecimentos (Torres; Do Amaral, 2011).

Pesquisas desenvolvidas por Lopes (2007), Pimentel, Fuks e Lucena (2008), Cunha (2009), Cardoso (2010), Gianotto e Diniz (2010), Oliveira e Tedesco (2010), Torres e Do Amaral (2011), Massaro (2014), dentre outros, abordam o uso da CSCL como uma estratégia educativa capaz de apresentar mais e melhores condições ao aluno para participar ativamente do processo de construção de seu próprio conhecimento e do grupo como um todo.

No cenário colaborativo, torna-se fundamental a presença de instrumentos, sejam eles informatizados ou não, que possibilitem mensurar e avaliar a colaboração de cada aluno, bem como do grupo como um todo, de modo que seja possível identificar, por exemplo, se houve colaboração, se as informações compartilhadas foram significativas e se os instrumentos contribuíram, de alguma forma, para a aprendizagem dos envolvidos.

O Collabora é um objeto virtual de aprendizagem colaborativa, fundamentado na CSCL, que permite maior agilidade no processo de obtenção de dados sobre a aprendizagem referente ao indivíduo ou ao grupo e promove uma participação mais significativa desses dados, tornando-os assim, participantes ativos do processo de ensino e aprendizagem para aquisição do conhecimento estatístico e probabilístico (Ishikawa et al., 2016).

O Collabora foi usado como ferramenta de apoio à disciplina de Probabilidade e Estatística. Por meio desta ferramenta o aluno pode construir, interagir e colaborar com os seus pares, podendo criar estratégias para solucionar problemas durante a resolução dos 
exercícios de uma atividade e, após a submissão da resposta consensual do grupo, obter retorno automático das respostas, o que auxilia na correção e possíveis ajustes dessas estratégias. Ao utilizar o objeto, o professor tem acesso a uma ferramenta que permite gerenciar disciplinas, além de outras atividades e obter a nota de colaboração do indivíduo ou do grupo.

Este artigo apresenta os dados obtidos com o uso do objeto educacional, analisados usando Análise Textual Discursiva (ATD) (Moraes; Galiazzi, 2016), em que se criou a categoria Aprendizagem Colaborativa com o objetivo de investigar as contribuições da aprendizagem colaborativa no processo de ensino e aprendizagem, considerando as áreaschaves da CSCL (Comunicação, Coordenação, Colaboração e Percepção).

\section{Referencial Teórico}

A Aprendizagem Colaborativa Apoiada por Computadores (CSCL) é a ciência que estuda como as pessoas podem aprender em grupo com o auxílio do computador, podendo ser aplicada em todos os níveis da educação formal, desde o jardim de infância até a graduação (STAHL et al., 2006).

Considerando a complexidade do desenvolvimento e aplicação CSCL, Otsuka (1999); Fuks e Assis (2001); Caballé et al. (2004); Cunha (2009); Cardoso (2010) descreveram funcionalidades que um sistema CSCL deve reunir, a fim de apoiar as atividades colaborativas. Dentre as principais áreas-chave da CSCL destacam-se: Comunicação, Coordenação, Colaboração e Percepção.

É durante a Comunicação que ocorrem as trocas de ideias, discussões e conflitos entre os pares (Otsuka, 1999). Para tanto, utiliza-se de ferramentas de comunicação mediadas por computador, síncronas ou assíncronas, que dão suporte às interações entre os participantes e que, normalmente, envolvem negociação.

A Coordenação envolve tanto a pré-articulação das tarefas, por meio de ações necessárias para preparar a colaboração antes do trabalho colaborativo se iniciar, seu gerenciamento, e a pós-articulação que ocorre ao final das tarefas, como um processo de avaliação sobre o que foi feito (Cardoso, 2010). Essa está diretamente relacionada com a avaliação colaborativa, que tem como principal atividade auxiliar na representação e construção colaborativa dos conhecimentos, pois permite que os resultados das atividades sejam avaliados, discutidos e aperfeiçoados, gerando novos conhecimentos compartilhados pelo grupo.

A área-chave de Colaboração pressupõe a ação de compartilhamento, uma vez que, ao partilharem objetos, informações e ideias, todos os participantes terão acesso aos artefatos e decisões compartilhadas pelo grupo.

A Percepção encontra-se no centro do processo colaborativo, uma vez que a mesma provê um contexto para as atividades individuais e permite maior sinergia do grupo. Por meio de uma coordenação colaborativa, a percepção oferece ainda oportunidades para comunicações informais espontâneas e dá aos participantes respostas necessárias sobre o que se passa no recurso computacional colaborativo.

O Collabora é um objeto educacional construído com os princípios da CSCL e tem como propósito disponibilizar uma ferramenta que apoie o ensino e a aprendizagem dos conteúdos na disciplina de Probabilidade e Estatística no Ensino Superior e seja capaz de medir a colaboração dos alunos. Para tanto, disponibiliza um meio para a realização das atividades colaborativas, por meio de chat, links e compartilhamento de arquivos (Ishikawa et al, 2018).

A arquitetura do Collabora foi concebida para controlar as interações entre os participantes durante o desenvolvimento de atividades colaborativas. A arquitetura não visa apenas promover a colaboração, mas também avaliá-la para obter dados que possam 
ser analisados sob o aspecto da aprendizagem do indivíduo ou do grupo (Ishikawa et al, 2017).

Cabe ressaltar que todos os exercícios desenvolvidos e cadastrados no Collabora compõem o banco de questões produzido para uma disciplina, podendo, posteriormente, serem criados novos exercícios, os quais poderão ser incorporados ao banco de questões.

\section{Material e Método}

A Análise Textual Discursiva (ATD) tem como objetivo produzir novas compreensões sobre os fenômenos e discursos e tem a intenção de compreender e reconstruir conhecimentos existentes sobre os temas a serem investigados (Moraes; Galiazzi, 2016). A ATD está organizada em 4 (quatro) enfoques, sendo eles: desmontagem do texto (processo de unitarização); estabelecimento de relações (processo de categorização); captação do novo emergente (expressão das compreensões atingidas) e a auto-organização. O estabelecimento das relações ou processo dos 3 (três) primeiros, formam um ciclo que se constituem como elementos principais da metodologia ATD.

A análise dos dados apresentada neste artigo foi obtida por meio ATD e iniciou-se com a desmontagem dos textos (processo de unitarização), usada para realizar a delimitação do corpus de análise. O corpus foi constituído de 3 (três) instrumentos: diário de bordo $^{1}$; anotações das atividades presenciais em grupo e dos registros (interações, troca de mensagens) produzidos pelos alunos no módulo Chat do Collabora.

As categorias, suas respectivas subcategorias e unidades foram identificadas por meio da fundamentação teórica sobre CSCL, relatadas na Seção 2. Também foram considerados alguns excertos coletados durante as aulas da disciplina e do uso do Collabora, os quais permitiram indicar contribuições do uso do objeto educacional proposto na disciplina no contexto da CSCL.

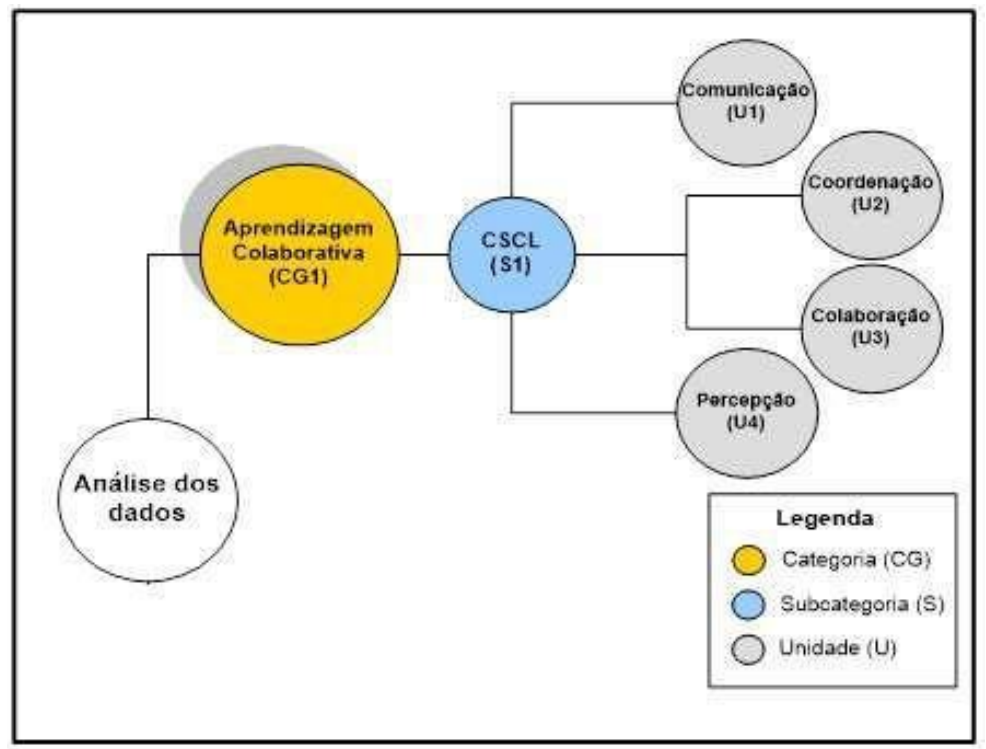

Figura 1 - Visão Geral da estrutura de análise dos dados.

Tendo como objetivo sintetizar o volume de dados em cada categoria de análise, foram selecionadas algumas das frases (excertos) avaliados como mais representativos em relação aos outros e, na sequência, foram informados os seus indicadores simbólicos. Para a organização e compreensão dos dados, foram utilizadas codificações apresentadas no quadro 1.

\footnotetext{
1 O Diário de bordo é o material no qual constam anotações fiéis do processo de acompanhamento das aulas da disciplina em que foi realizado a análise e o exceto das atividades em grupo.
} 
Quadro 1 - Decodificação.

\begin{tabular}{|l|l|}
\hline \multicolumn{1}{|c|}{ Descrição } & \multicolumn{1}{c|}{ Indicadores simbólicos } \\
\hline Alunos & $\mathrm{A} 21, \ldots, \mathrm{A} 63$ \\
\hline Grupos & $\mathrm{G} 2, \mathrm{G} 2, \mathrm{G} 4, \ldots, \mathrm{G} 12$ \\
\hline Chat do Collabora & $\mathrm{C} 1{ }^{2}, \mathrm{C} 2, \ldots \mathrm{C} 10$ \\
\hline Atividade do Collabora & $\mathrm{ATV}$ \\
\hline Diário de bordo & $\mathrm{DB}$ \\
\hline Atividades presenciais em grupo & $\mathrm{APG}$ \\
\hline Categoria & $\mathrm{CG} 1, \mathrm{CG} 2$ \\
\hline Subcategoria & $\mathrm{S} 1, \mathrm{~S} 2$ e S3 \\
\hline Unidade & $\mathrm{U} 1, \mathrm{U} 2, \mathrm{U} 3, \mathrm{U} 4, \mathrm{U} 5, \mathrm{U} 6, \mathrm{U} 7, \mathrm{U} 8 \mathrm{E}$ U9 \\
\hline
\end{tabular}

Nos casos do Diário de Bordo (DB) e das Atividades presenciais em grupo (APG), constará na sequência de seus indicadores simbólicos a Linha (L) na qual se encontra o excerto. Nesse caso, tem-se como exemplo de codificação de excerto: A21.G2.DB.L20 ${ }^{3}$ (Aluno21, pertencente ao Grupo2, coletado no Diário de bordo encontrado na Linha 20), seguido da Categoria, Subcategoria e Unidade quando forem necessários.

Para o Chat, constarão na codificação dos excertos, além do(s) aluno (s) e grupo, a atividade a que pertencem os diálogos. Tem-se como exemplo: A22;A23;A24.G3.C.ATV1 (Aluno22; Aluno23; Aluno24 que pertencentes ao Grupo3, coletado no Chat do Collabora na Atividade 1).

\section{Resultados e Discussões}

Os resultados descritos com o uso do Collabora foram obtidos por meio de um experimento realizado na disciplina de Probabilidade Estatística de uma Universidade Federal no qual participaram 11 grupos de 3 a 4 participantes, totalizando 41 alunos do curso de graduação e cada grupo fez um total de 27 exercícios, distribuídos em 10 (dez) atividades.

Conforme ilustrado na figura 1 , foi configurada a categoria (CG1), denominada "Aprendizagem Colaborativa", e esta foi criada em função do interesse de se investigar as contribuições de um objeto de aprendizagem (Collabora) na aprendizagem relacionada as área-chaves da CSCL. As unidades de análise estão apresentadas no quadro 2.

Quadro 2 - Descrição das unidades de análise da CG1.

\begin{tabular}{|c|c|c|}
\hline CATEGORIA & & SUBCATEGORIAS E UNIDADES \\
\hline \multirow{4}{*}{$\begin{array}{l}\text { CG1 - } \\
\text { Aprendizagem } \\
\text { Colaborativa }\end{array}$} & \multirow{4}{*}{$\begin{array}{l}\text { S1 - CSCL } \\
\text { (Computer } \\
\text { Supported } \\
\text { Collaborative } \\
\text { Learning). }\end{array}$} & $\begin{array}{l}\text { U1 - Comunicação } \\
\text { Foram classificados em CG1.S1.U1 os excertos dos alunos que } \\
\text { evidenciaram trocas de ideias por meio do Collabora. }\end{array}$ \\
\hline & & $\begin{array}{l}\text { U2 - Coordenação } \\
\text { Foram classificados em CG1.S1.U2 os excertos dos alunos que } \\
\text { demonstraram: formação de equipe, definição e planejamento dos } \\
\text { objetivos, acompanhamento das atividades, verificação de: } \\
\text { resultados, prazo, escopo e recursos. }\end{array}$ \\
\hline & & $\begin{array}{l}\text { U3 - Colaboração } \\
\text { Foram classificados em CG1.S1.U3 os excertos dos alunos que } \\
\text { demonstraram: negociação entre os pares e votação de propostas. }\end{array}$ \\
\hline & & $\begin{array}{l}\text { U4 - Percepção } \\
\text { Foram classificados em CG1.S1.U4 os excertos dos alunos que } \\
\text { demonstraram informações sobre o que se passava no Collabora, tais } \\
\text { como: trocas de informações referente às atividades, tarefas, tempo, } \\
\text { feedbacks, entre outros. }\end{array}$ \\
\hline
\end{tabular}

\footnotetext{
${ }^{2} \mathrm{C} 1$ refere-se ao Chat da atividade 1.

${ }^{3}$ As referências das codificações dos excertos utilizam a separação por ponto (.).
} 
As 4 (quatro) unidades descritas na CG1 foram estabelecidas por excertos coletados durante a prática das 10 (dez) atividades realizadas pelos grupos por meio do Chat do Collabora.

Apresenta-se, a seguir, um exemplo de excerto categorizado na CG1.S1.U1 o qual evidenciou a existência da unidade de análise Comunicação (U1), conforme ilustrado no quadro 3.

\section{Quadro 3 - Unidade de análise Comunicação (U1).}

[...] A58-[...] A está certa, acabei de ver na apostila

A44 - blz

A57 - entreii

A57 - a está certa mesmo

A55 - beleza kk

A58 - pelo que pesquisei a B são exemplos de um erro não amostral

A58 - calmai A58 - achei isso

A57 - pelo q pesquisei [...] Um erro amostral é a diferença entre o resultado amostral e o verdadeiro resultado da população; tais erros resultam das flutuações amostrais devidas ao acaso. Um erro não amostral ocorre quando os dados amostrais são coletados, registrados ou analisados incorretamente (tal como a seleção de uma amostra tendenciosa, o uso de um instrumento de medida defeituoso, ou cópia incorreta dos dados).

A44 - então pelo que vi sim

A58 - exatamente isso que A57 mandou [...] (A44;A55;A57;A58.G6.C.ATV2).

O excerto A44;A55;A57;A58.G6.C.ATV2 demonstra a comunicação entre os alunos durante a realização da segunda atividade disponibilizada para essa equipe no Collabora, a qual tratava dos conteúdos de Visão Geral e Medidas de Tendência Central. Nota-se, neste excerto, que um dos alunos buscou informações na apostila e utilizou o Chat do Collabora para trocar ideias com os demais membros do grupo, efetivando um diálogo que favorece a aprendizagem colaborativa.

Percebeu-se que o Collabora facilitou a comunicação interativa entre os membros do grupo, pois, conforme já apontado por Crook (1996), Cortelazzo (2000), Torres e Do Amaral (2011), os recursos computacionais favoreceram o processo de aprendizagem colaborativa, pois possibilitaram gerar um processo comunicacional interativo, dinâmico e bilateral entre os envolvidos. A segunda unidade de análise a Coordenação (U2), possui excertos categorizados na CG1.S1.U2, que foram selecionados por representarem o planejamento de objetivos, a verificação de resultados, o acompanhamento do tempo e/ou prazo das atividades, conforme ilustrados no quadro 4.

Quadro 4 - Unidade de análise Coordenação (U2).

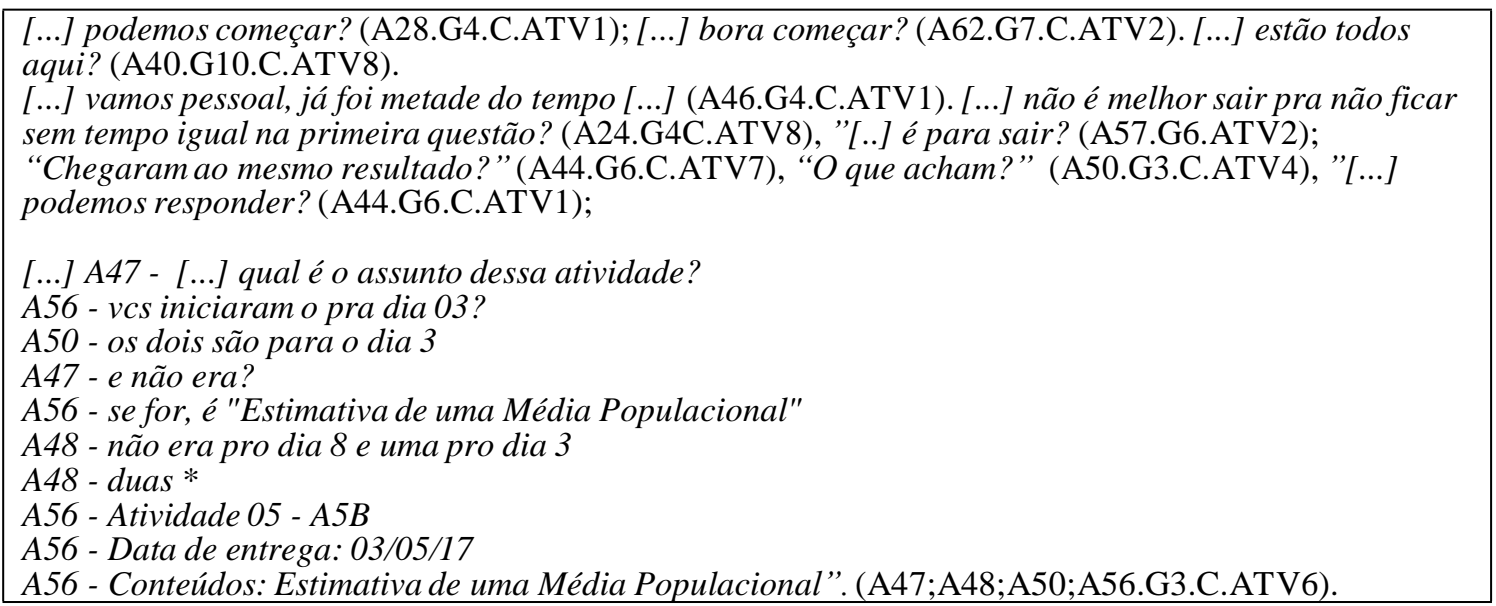

Os excertos apresentados no quadro 4 ratificam a importância da unidade de análise Coordenação (U2) durante o processo de construção da aprendizagem 
colaborativa. Conforme descrito por Cardoso (2010), a coordenação envolve a préarticulação das tarefas (ações necessárias para preparar a colaboração), seu gerenciamento (durante o processo) e, finalmente, a pós-articulação (avaliação sobre o que foi feito).

É possível perceber que existe uma pré-articulação das tarefas, no qual pelo menos um dos integrantes do grupo procura iniciar o trabalho colaborativo, indagando se todos estavam prontos para iniciar a atividade (A28.G4.C.ATV1; A62.G7.C.ATV2), ou se a equipe estava completa antes de começarem as discussões no chat (A40.G10.C.ATV8). Durante o desenvolvimento da atividade (gerenciamento), observa-se que existe a preocupação dos alunos em administrar os recursos disponíveis (tempo) e resultados esperados (A46.G4.C.ATV1; A24.G4C.ATV8).

Ao final do processo (pós-articulação), nota-se que ocorre a avaliação e o grupo percebeu a necessidade de se comunicar, tomar decisões em conjunto e rever resultados, conforme já estabelecido por Pimentel, Fuks e Lucena (2008) e demonstrados pelos excertos A44.G6.C.ATV7, A50.G3.C.ATV4 e A44.G6.C.ATV1.

Da mesma forma, o diálogo dos alunos apresentado no excerto A47; A48; A50;A56.G3.C.ATV6 é um exemplo de coordenação do G3, no qual o grupo discute a respeito das tarefas antes de iniciar o trabalho colaborativo, ou seja, a execução das atividades da disciplina previstas no objeto. A discussão ocorre em torno dos prazos de entrega das atividades e do conteúdo abordado na mesma. Esse era um aspecto importante para os alunos, uma vez que as atividades no Collabora tinham conteúdos e datas de entrega pré-estabelecidas. O não cumprimento dos prazos inviabilizava, de forma automática, o envio das atividades pelo grupo.

Os excertos categorizados na CG1.S1.U3, apresentados no quadro 5, comprovam a existência da unidade de análise Colaboração (U3) e mostram a existência de negociação e votação de propostas entre os pares.

Quadro 5 - Unidade de análise Colaboração (U3).

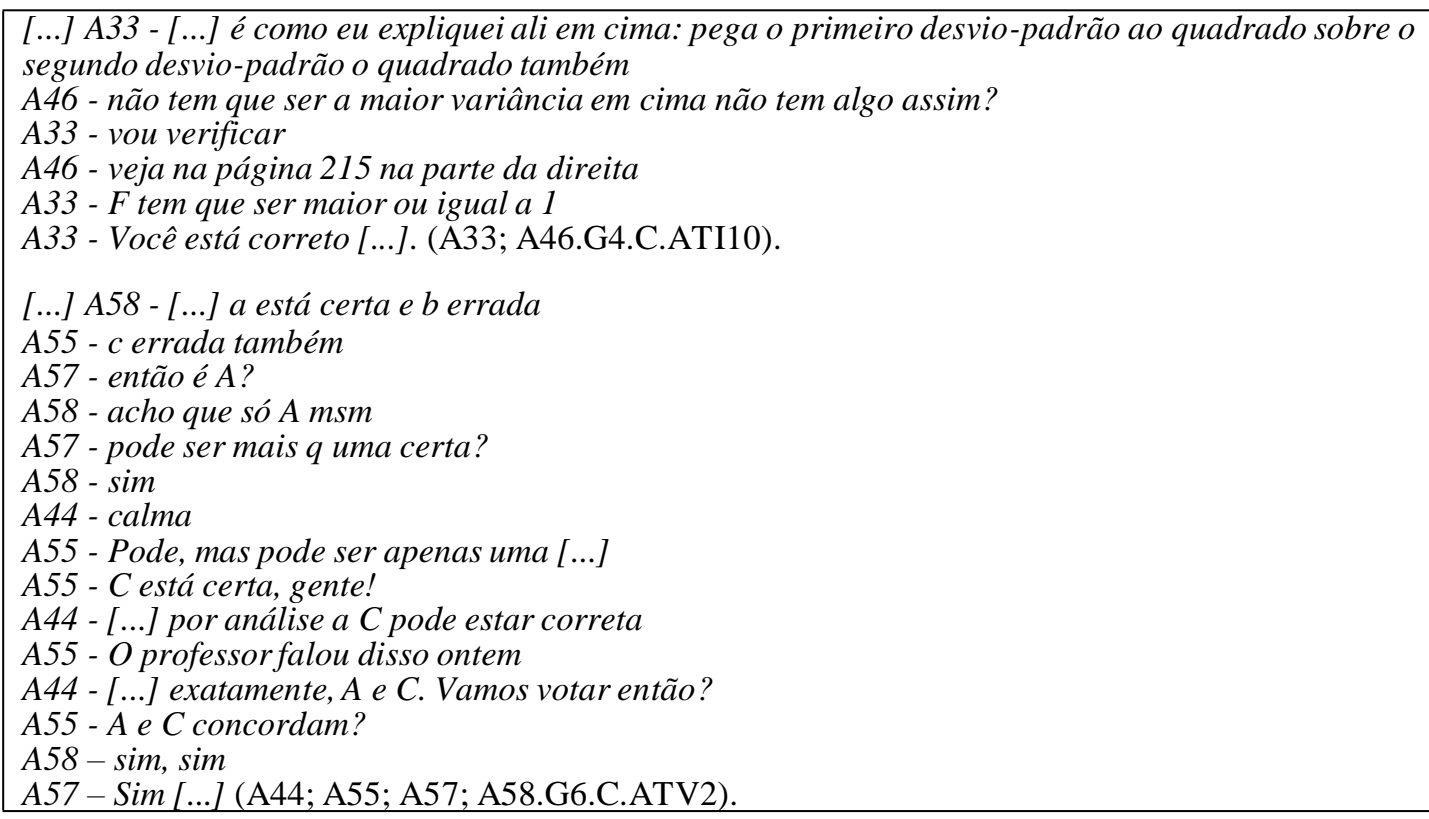

O excerto categorizado A33.A46.G4.C.ATI10 apresentou um diálogo entre os alunos A33 e A46, no qual é possível identificar um conflito de informações entre eles acerca do conteúdo de comparação de duas variâncias. Pautado nos conhecimentos adquiridos nas aulas e no conteúdo da apostila, o aluno A46 obteve argumentos apropriados que permitiu ao seu colega A33 do grupo, um maior entendimento dos conceitos relacionados à atividade de teste de hipóteses. 
Ainda no quadro 5, é possível identificar no excerto A44.A55.A57. A58.G6.C.ATV2, a presença da atividade de negociação entre os alunos. Nota-se uma colaboração ativa, em que a avaliação dos resultados por todos os membros do grupo é imperativa. Diante da necessidade de se tomar uma decisão em conjunto, o Collabora forneceu recursos que permitiram a resolução de conflitos, por meio da negociação de propostas entre os participantes e que, após as argumentações do grupo, os alunos partiram para a tomada de decisão e realizaram a votação de sugestões conflitantes, conforme já exposto por Otsuka (1999; 2005), ao elencar a obrigatoriedade dessas soluções para sistemas CSCL.

O compartilhamento de informações ou a resposta à dúvida de outros integrantes possibilitaram a compreensão e a concordância do grupo pela resolução de uma atividade. Entende-se, portanto, como já exposto por Torres (2007), que essa troca de informações, ideias e decisões compartilhadas pelo grupo, levam à aprendizagem dos alunos de modo colaborativo. Cabe ressaltar que o Collabora forneceu suporte para que os alunos apresentassem e discutissem suas opiniões, argumentos e tomassem uma decisão que satisfizesse a maioria por meio de um processo de votação automática.

Os excertos categorizados na unidade de análise Percepção (U4) descritos no quadro 6, mostraram as dificuldades e dúvidas apresentadas pelos alunos durante a utilização do Collabora.

Quadro 6 - Unidade de análise Percepção (U4).

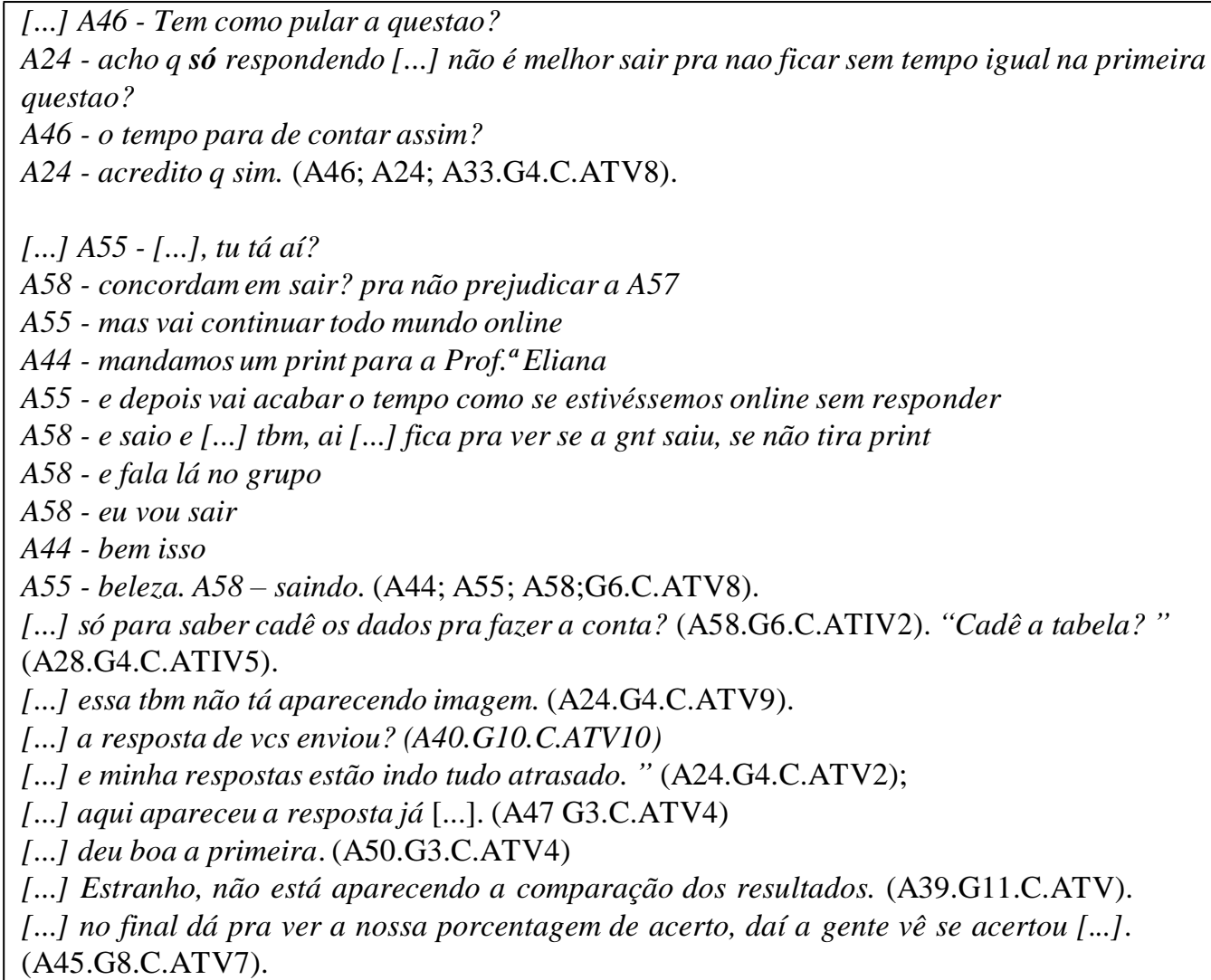

Os registros A46; A24; A33.G4.C.ATV8 e A44; A55; A58;G6.C.ATV8, evidenciaram as dificuldades dos alunos em relação à ordem de execução dos exercícios e do tempo permitido para a realização da atividade. Além disso, percebe-se que o G6 está em dúvida se o tempo da atividade irá mesmo parar quando todos os integrantes estiverem off-line, assim, relatam que irão "tirar um print" da tela e entrarão em contato com o responsável pelo Collabora para sanar a dúvida. 
Os demais excertos se referem às dúvidas ou problemas percebidos pelos alunos durante a execução das atividades no Collabora, alguns relacionados a problemas ou dúvidas dos enunciados e/ou dados dos exercícios, outros referentes ao retorno (feedback) do objeto, após a finalização de uma atividade.

É possível verificar que alguns grupos apresentaram dificuldades em compreender o funcionamento do Collabora. Em alguns casos, os grupos efetuavam o login, resolviam os exercícios e quando retornavam, em outro momento, para terminar a atividade, o tempo total já havia expirado. Isso pode ter ocorrido pelo fato de ainda haver pelo menos dois integrantes conectados ou problemas técnicos (programação, conexão e internet), porém, cada caso, foi acompanhado durante a aplicação da ferramenta.

Pressupõe-se que a percepção presente no Collabora permitiu aos grupos realizar uma coordenação implícita da aprendizagem colaborativa. Corroborando com Caballé et al. (2004), a ferramenta ofereceu oportunidades para comunicações espontâneas e deu aos participantes respostas necessárias sobre tudo o que estava acontecendo no ambiente. Percebe-se que houve uma coordenação dos próprios participantes, aumentando a sua responsabilidade em relação às informações geradas, de forma que cada um estivesse ciente sobre os eventos que ocorriam no objeto colaborativo, conforme já mencionado por Cunha (2009).

\section{Considerais finais}

Este artigo analisou as contribuições de um objeto educacional no contexto da CSCL. Em relação a subcategoria de Comunicação foi possível perceber que o Collabora permitiu a comunicação síncrona entre os alunos durante a realização das atividades disponibilizadas. A ferramenta chat deu suporte às interações e promoveu uma maior proximidade 'virtual' entre os participantes, que puderam trocar ideias, discutir e resolver eventuais conflitos nos grupos de trabalho, efetivando assim, um diálogo que favoreceu a comunicação interativa e a aprendizagem colaborativa.

$\mathrm{Na}$ subcategoria Coordenação foi possível constatar que, em alguns grupos de trabalho houve coordenação, pois as tarefas foram definidas e organizadas para que efetivamente ocorresse a colaboração. Nesses grupos, percebeu-se que as principais etapas da coordenação se fizeram presentes, iniciando pela pré-articulação das tarefas, passando pelo gerenciamento e sendo finalizadas na pós-articulação. Conforme visto nos excertos, os alunos procuraram dar início ao trabalho colaborativo, indagando se todos estavam prontos para iniciar a atividade ou se a equipe estava completa antes de iniciarem as discussões no chat. Além disso, existia a preocupação constante em se administrar os tempos (prazos de entrega) e avaliar os resultados.

Considerando os diálogos e as interações apresentadas nos excertos em relação a unidade Colaboração foi possível observar a colaboração entre os integrantes nos grupos de trabalho. A ação de compartilhamento, disseminação e troca de recursos entre os aprendizes possibilitou que ocorresse o processo de negociação, sendo essa uma das principais características de uma atividade colaborativa e, na sequência, a votação de propostas. Os conflitos de informações entre os alunos, acerca dos conteúdos estatísticos e a troca de informações, seja por meio dos conhecimentos adquiridos nas aulas ou pesquisas na internet, permitiram um maior entendimento dos conceitos relacionados às atividades propostas.

Os excertos em relação a Percepção apontam as dificuldades e dúvidas apresentadas pelos alunos durante a utilização do Collabora. As principais dificuldades dos alunos foram em relação à ordem de execução dos exercícios e do tempo permitido para a realização da atividade. As dúvidas ou problemas percebidos pelos alunos estavam relacionados a problemas ou dúvidas do exercício, seja no enunciado ou ao retorno 
(feedback) do objeto, após a finalização de uma atividade. Os relatos dos alunos apontam a falta de conhecimento da ferramenta e das regras de execução das atividades, além disso, eventuais problemas técnicos da própria ferramenta (programação, conexão e internet) ou elaboração do exercício, porém, cada caso foi acompanhado durante a aplicação da ferramenta.

Em síntese, o Collabora cumpriu com os requisitos mínimos de um sistema CSCL, apoiou o processo colaborativo e deu suporte para que os grupos trocassem informações, discutissem entre si e tomassem decisões em conjunto. A ferramenta auxiliou e permitiu que os participantes apresentassem suas opiniões, resolvessem os conflitos durante a tomada de decisões, de forma que satisfizesse a maioria por meio da negociação e um processo de votação automática. Entende-se, portanto, que essa troca de informações, ideias e decisões compartilhadas pelo grupo levam à aprendizagem dos alunos de modo colaborativo.

\section{Referências Bibliográficas}

CABALLÉ, S. et al. Towards a Generic Platform To Developing CSCL Applications Using Grid Infrastructure. IEEE International Symposium on Cluster and the Grid. 2004.

CARDOSO, A. L. M. de S. Construção e difusão colaborativa do conhecimento: uma experiência construtivista de educação em um ambiente virtual de aprendizagem. 2010. 305 f. Tese (Doutorado em Educação) - Universidade Federal da Bahia, Salvador, 2010.

CORTELAZZO, I. B. C. Colaboração, trabalho em equipe e as tecnologias de comunicação: relações de proximidade em cursos de pós-graduação. 2000. 210f. Tese (Doutorado em Educação) - Faculdade de Educação, Universidade de São Paulo, São Paulo, 2000.

CROOK, C. Computers and the collaborative experience of learning. New York: Routledge, 1996.

CUNHA, A. A. S. da. Definição de um modelo de sistema de aprendizagem colaborativa apoiado por computador com ênfase em trabalho em equipe 2009. 146f. Dissertação (Mestrado em Engenharia Elétrica e de Computação) - Universidade Estadual de Campinas. Campinas, 2009.

FUKS, H; ASSIS, R.L. Facilitating perception on virtual learningware based environments, The Journal of Sustem and Information Technology, v.5., n. 01, Edith Cowan University, Australia, p. 93-113, 2001.

GIANOTTO, D. E. P.; DINIZ, R. E. DA S. Formação inicial de professores de Biologia: a metodologia colaborativa mediada pelo computador e a aprendizagem para a docência. Ciência \& Educação, Bauru, v. 16, n. 3, p. 631-648, 2010.

GOMES, E. R.; Silveira, R. A.; Viccari, R. M. Objetos Inteligentes de Aprendizagem: Uma Abordagem baseada em Agentes para Objetos de Aprendizagem. In: Simpósio Brasileiro de Informática na Educação. XV., 2004, Manaus. Anais... Manaus: UFAMAM, 2004. p.389-398.

ISHIKAWA, E. C M.; NOVELLI, L.; MATOS, S. N.; ANDRADE, V.; SANTOS JÚNIOR, G. Modelo Conceitual do Objeto Virtual de Aprendizagem Colaborativa para Aprendizagem de Estatística (Collabora). RENOTE, v. 14, n. 2, P. 1 - 10; 2016. 
ISHIKAWA, E. C. M.; BELUZZO, L. B.; MATOS. S. N.; SANTOS JÚNIOR, G. . Collabora: A Colaborative Architecture for Evaluating Individuals Participation During the Development of Activities. International Journal of Software Engineering \& Applications (IJSEA), v. 8, p. 33-48, 2017.

LOPES, M. S. S. Avaliação da aprendizagem em atividades colaborativas em EaD viabilizada por um fórum categorizado. 2007. 168f. Dissertação (Mestrado em Informática) - Universidade Federal do Rio de Janeiro, Instituto de Matemática, Núcleo de Computação Eletrônica, 2007.

MASSARO, G. Recursos Educacionas abertos e aprendizagem colaborativa: novas perspectivas na construção e utilização de materias educacionais. Colabor@-A Revista Digital da CVA-RICESU, v. 8, n. 31, 2014.

MORAES, Roque; GALIAZZI, Maria do Carmo. Análise Textual Discursiva. $3^{\text {a }}$ ed. Editora Unijuí, Ijuí, 2016.

OLIVEIRA, E. A.; TEDESCO, P. i-collaboration: Um modelo de colaboração inteligente personalizada para ambientes de EAD. Revista Brasileira de Informática na Educação, v. 18, n. 1, p. 17-31, 2010.

OTSUKA, Joice Lee. SAACI - Sistema de Apoio à Aprendizagem Colaborativa na Internet. 1999. 127 f. Dissertação (Mestrado em Computação) - Programa de PósGraduação em Computação - Universidade Federal do Rio Grande do Sul, Porto Alegre, 1999.

OTSUKA, Joice Lee; DA ROCHA, Heloísa Vieira. Um modelo de suporte à avaliação formativa para ambientes de educação à distância: dos conceitos à solução tecnológica. RENOTE, v. 3, n. 2, 2005.

PIMENTEL, M.; FUKS, H.; LUCENA, C.JP. Um processo de desenvolvimento de sistemas colaborativos baseado no Modelo 3C: RUP-3C-Groupware. In: Simpósio Brasileiro de Sistemas de Informação. IV., 2008, Rio de Janeiro. Anais...Rio de Janeiro, 2008. p. 35-47.

STAHL, G., et al. Computer-supported collaborative learning: An historical perspective. Cambridge handbook of the learning sciences, 2006.

TORRES, P. L. Aprendizagem colaborativa. Algumas vias para entretecer o pensar e o agir. Curitiba: SENAR-PR, p. 65-98, 2007.

TORRES, T. Z.; DO AMARAL, S. F. Aprendizagem Colaborativa e Web 2.0: proposta de modelo de organização de conteúdos interativos. Educação Temática Digital, v. 12, p. 49, 2011. 